\title{
A Reaction-Diffusion System of a Predator-Prey-Mutualist Model
}

\author{
SINING ZHENG \\ Department of Mathematics, University of Michigan, Ann Arbor, Michigan 48109 \\ and Department of Applied Mathematics, Dalian Institute of Technology, \\ Dalian, People's Republic of China*
}

Received 22 March 1985; revised 5 September 1985

\begin{abstract}
Mutualism is part of many significant processes in nature. Mutualistic benefits arising from modification of predator-prey interactions involve interactions of at least three species. In this paper we investigate the homogeneous Neumann problem and Dirichlet problem for a reaction-diffusion system of three species--a predator, a mutualist-prey, and a mutualist. The existence, uniqueness, and boundedness of the solution are established by means of the comparison principle and the monotonicity method. For the Neumann problem, we analyze the constant equilibrium solutions and their stability. For the Dirichlet problem, we prove the global asymptotic stability of the trivial equilibrium solution. Specifically, we study the existence and the asymptotic behavior of two nonconstant equilibrium solutions. The main method used in studying of the stability is the spectral analysis to the linearized operators. The O.D.E. problem for the same model was proposed and studied in [13]. Through our results, we can see the influences of the diffusion mechanisin and the different boundary value conditions upon the asymptotic behavior of the populations.
\end{abstract}

\section{INTRODUCTION}

Mutualism is part of many significant systems and processes, such as mycorrhizal associations, nitrogen fixation, gut faunas and floras, endosymbiotic photosynthesis, endozoic algae, etc. A interaction among organisms of different species is called mutualistic if the presence of each species enhances the per capita growth rate of the other.

Mutualistic benefits arising from modification of predator-prey or competitive interactions involve interactions of at least three species. A mutualist may affect a predator-prey interaction to the benefit of either the predator or the prey, but the most common pattern is a mutualist deterring predation on

* Permanent address. 
a prey. For example, ants deter herbivores from feeding on plants and deter predators from feeding on aphids, endozoic algae deter predators from feeding on protozoans, and crustacea deter starfish from feeding on corals.

In [13], B. Rai, H. I. Freedman, and J. F. Addicott presented two models of mutualism among three species and analyzed the conditions for boundedness of solutions, the equilibria and their local stability, and the condition for the existence of small amplitude periodic solutions. One of the two models involves interaction among a predator $(y)$, a mutualist-prey $(x)$, and a mutualist $(u)$. A special case examined in some detail by the authors of [13] is the following O.D.E. problem:

$$
\begin{aligned}
& \frac{d u}{d t}=\gamma u\left(1-\frac{u}{L_{u}+l x}\right), \\
& \frac{d x}{d t}=\alpha x\left(1-\frac{x}{K}\right)-\frac{\beta x y}{1+m u}, \\
& \frac{d y}{d t}=y\left(-s+\frac{c \beta x}{1+m u}\right),
\end{aligned}
$$

where $\gamma, L_{0}, l, \alpha, K, \beta, m, s, c$ are all positive parameters, $l$ and $m$ being the mutualism constants. The model (1.1) has the following features:

(1) The mutualist deters predation on the prey.

(2) The mutualist growth is of logistic type.

(3) The prey growth in the absence of predator is logistic and independent of the mutualist, i.e., the prey is benefitted by the mutualist only when the predator is present.

(4) Predation in the absence of mutualism is Lotka-Volterra predation.

It is known that the distributions of populations, in general, being heterogeneous, depend not only on time, but also on the spatial positions in the habitat. So it is natural and more precise to study the corresponding P.D.E. problem.

In this paper, taking account of the diffusion mechanism, we consider the corresponding nonlinear reaction-diffusion system

$$
\begin{aligned}
& \frac{\partial u_{1}}{\partial t}=d_{1} \Delta u_{1}+\gamma u_{1}\left(1-\frac{u_{1}}{L_{0}+l u_{2}}\right), \\
& \frac{\partial u_{2}}{\partial t}=d_{2} \Delta u_{2}+\alpha u_{2}\left(1-\frac{u_{2}}{K}\right)-\frac{\beta u_{2} u_{3}}{1+m u_{1}}, \text { in } \Omega \times \mathbb{R}^{+}, \\
& \frac{\partial u_{3}}{\partial t}=d_{3} \Delta u_{3}+u_{3}\left(-S+\frac{c \beta u_{2}}{1+m u_{1}}\right)
\end{aligned}
$$


with initial condition

$$
u_{i}(x, 0)=u_{i 0}(x), \quad i=1,2,3, \quad \text { on } \Omega,
$$

and homogeneous Neumann boundary condition

$$
\frac{\partial u_{i}}{\partial n}=0, \quad i=1,2,3, \quad \text { on } \partial \Omega \times \mathbb{R}^{+},
$$

or homogeneous Dirichlet boundary condition

$$
u_{i}(x, t)=0, \quad i=1,2,3, \quad \text { on } \partial \Omega \times \mathbb{R}^{+},
$$

where $u_{1}, u_{2}$, and $u_{3}$ represent the populations of mutualist, prey, and predator with diffusion constants $d_{1}, d_{2}$, and $d_{3}$, respectively; $\Omega$ is a bounded domain in $\mathbb{R}^{n} ; \partial \Omega$ is the boundary of $\Omega ; \partial / \partial n$ represents the outward normal derivatives on $\partial \Omega$; and $\Delta$ is the Laplace operator.

The homogeneous Neumann boundary value condition (1.4) is to be interpreted as a "no flux" condition (i.e., there is no migration of any of the populations across the boundary of their habitat), whereas the homogeneous Dirichlet condition is to be considered as a condition under which none of the three species can exist on the boundary of the habitat.

We establish the existence, uniqueness, and boundedness of the solution for the both kinds of boundary value problems by means of the comparison principle and the monotonicity method (e.g., $[8,9,12,15])$. For the Neumann problem, we analyze the constant equilibrium solutions and their asymptotic stability. For the Dirichlet problem, we give the condition under which the trivial equilibrium solution is asymptotically stable, i.e., all populations go to extinction. Specifically, we study the existence and the asymptotic stability of two nonconstant equilibrium solutions (the mutualist or the prey is alive alone as time tends to infinity). The main method used in studying the stability is the spectral analysis of the linearized operators $[6-9,11]$.

Comparing our results with those of [13], one can see clearly the influences of the diffusion mechanism and the different boundary value conditions upon the asymptotic behavior of the populations.

For a detailed explanation of the ecological background of the problem, the reader is referred to [13].

\section{PRELIMINARIES}

In this section we introduce the concept of upper and lower solutions as well as the existence-comparison theorem, which will be very useful to us in establishing the existence, uniqueness, and boundedness, and even in studying the asymptotic behavior (in some cases), of the solutions. 
We first consider the more general system

$$
\begin{aligned}
& \frac{\partial u_{1}}{\partial t}-L_{1} u_{1}=f_{1}\left(u_{1}, u_{2}\right), \\
& \frac{\partial u_{2}}{\partial t}-L_{2} u_{2}=f_{2}\left(u_{1}, u_{2}, u_{3}\right), \\
& \frac{\partial u_{3}}{\partial t}-L_{3} u_{3}=f_{3}\left(u_{1}, u_{2}, u_{3}\right)
\end{aligned}
$$

with boundary condition

$$
B_{i}\left[u_{i}\right]=\alpha_{i}(x) u_{i}+\beta_{i}(x) \frac{\partial u_{i}}{\partial n}=h_{i}(x), \quad i=1,2,3, \quad \text { on } \partial \Omega \times \mathbb{R}^{+}
$$

and initial condition

$$
u_{i}(x, 0)=u_{i 0}(x), \quad i=1,2,3, \quad \text { on } \Omega,
$$

where $L_{i}$ is an uniformly elliptic operator in $\Omega, i=1,2,3$.

Assume that $\alpha_{i}, \beta_{i}$, and $u_{i 0}$ are smooth nonnegative functions with $u_{i 0} \neq 0, \alpha_{i}+\beta_{i}>0$, and that $f_{i}$ is continuously differentiable with respect to its variables for $u_{k} \geqslant 0, i, k=1,2,3$. In addition, we require the following assumptions on $f_{i}, i=1,2.3$ :

$$
\begin{aligned}
& \frac{\partial f_{1}}{\partial u_{2}} \geqslant 0, \\
& \frac{\partial f_{2}}{\partial u_{1}} \geqslant 0, \quad \frac{\partial f_{2}}{\partial u_{3}} \leqslant 0, \text { for } \quad u_{i} \geqslant 0, i=1,2,3, \\
& \frac{\partial f_{3}}{\partial u_{1}} \leqslant 0, \quad \frac{\partial f_{3}}{\partial u_{2}} \geqslant 0
\end{aligned}
$$

which are natural in view of the ecological background of the predator-preymutualist model and satisfied both by the reaction terms of (1.2) and by those of a more general P.D.E. system corresponding to (2.1) of [13].

Now, we give the definition of the upper and lower solutions. DEFINITION 2.1

Ordered smooth functions $\bar{U}=\left(\bar{u}_{1}, \bar{u}_{2}, \bar{u}_{3}\right)$ and $\underline{U}=\left(\underline{u}_{1}, \underline{u}_{2}, \underline{u}_{3}\right)$ in $Q_{T}$ are called upper and lower solutions of (2.1)-(2.3), respectively, if they satisfy the 
following inequalities (2.5)-(2.7):

$$
\begin{gathered}
\left(\bar{u}_{1}\right)_{t}-L_{1} \bar{u}_{1} \geqslant f_{1}\left(\bar{u}_{1}, \bar{u}_{2}\right), \\
\left(\bar{u}_{2}\right)_{t} \quad L_{2} \bar{u}_{2} \geqslant f_{2}\left(\bar{u}_{1}, \bar{u}_{2}, \underline{u}_{3}\right), \\
\left(\bar{u}_{3}\right)_{t}-L_{3} \bar{u}_{3} \geqslant f_{3}\left(\underline{u}_{1}, \bar{u}_{2}, \bar{u}_{3}\right), \\
\left(\underline{u}_{1}\right)_{t}-L_{1} \underline{u}_{1} \leqslant f_{1}\left(\underline{u}_{1}, \underline{u}_{2}\right), \\
\left(\underline{u}_{2}\right)_{t}-L_{2} \underline{u}_{2} \leqslant f_{2}\left(\underline{u}_{1}, \underline{u}_{2}, \bar{u}_{3}\right) \\
\left(\underline{u}_{3}\right)_{t}-L_{3} u_{3} \leqslant f_{3}\left(\bar{u}_{1}, \underline{u}_{2}, \underline{u}_{3}\right) \\
B_{i}\left[\bar{u}_{i}\right] \geqslant h_{i}(x) \geqslant B_{i}\left(\underline{u}_{i}\right), \quad i=1,2,3, \quad \text { on } S_{T}, \\
\bar{u}_{i}(x, 0) \geqslant u_{i 0}(x) \geqslant \underline{u}_{i}(x, 0), i=1,2,3, \quad \text { on } \Omega,
\end{gathered}
$$

where $Q_{T}=\Omega \times(0, T], S_{T}=\partial \Omega \times(0, T]$, and $T<\infty$ but can be arbitrarily large.

Suppose $\bar{U}$ and $\underline{U}$ exist. Denote

$$
\begin{aligned}
\Sigma & =\left\{\left(u_{1}, u_{2}, u_{3}\right) \in \mathbb{R}^{3}: \underline{\rho}_{i} \leqslant u_{i} \leqslant \bar{\rho}_{i}, i=1,2,3\right\}, \\
M_{i} & =\sup _{\Sigma}\left\{\left|\frac{\partial f_{i}}{\partial u_{i}}\right|\right\}, \quad i=1,2,3,
\end{aligned}
$$

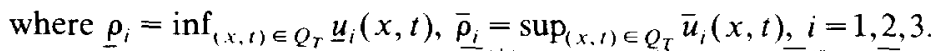

We construct the sequences $\left\{\bar{U}^{(k)}\right\}$ and $\left\{\underline{U}^{(k)}\right\}$ with $\bar{U}^{(0)}=\bar{U}$ and $\underline{U}^{(0)}=\underline{U}$ as follows:

$$
\begin{aligned}
& \left(\bar{u}_{1}^{(k)}\right)_{t}-L_{1} \bar{u}_{1}^{(k)}+M_{1} \bar{u}_{1}^{(k)}=M_{1} \bar{u}_{1}^{(k-1)}+f_{1}\left(\bar{u}_{1}^{(k-1)}, \bar{u}_{2}^{(k-1)}\right), \\
& \left(\bar{u}_{2}^{(k)}\right)_{t}-L_{2} \bar{u}_{2}^{(k)}+M_{2} \bar{u}_{2}^{(k)}=M_{2} \bar{u}_{2}^{(k-1)}+f_{2}\left(\bar{u}_{1}^{(k-1)}, \bar{u}_{2}^{(k-1)}, \underline{u}_{3}^{(k-1)}\right), \\
& \left(\bar{u}_{3}^{(k)}\right)_{t}-L_{3} \bar{u}_{3}^{(k)}+M_{3} \bar{u}_{3}^{(k)}=M_{3} \bar{u}_{3}^{(k-1)}+f_{2}\left(\underline{u}_{1}^{(k-1)}, \bar{u}_{2}^{(k-1)}, \bar{u}_{3}^{(k-1)}\right), \\
& \left(\underline{u}_{1}^{(k)}\right)_{t}-L_{1} \underline{u}_{1}^{(k)}+M_{1} \underline{u}_{1}^{(k)}=M_{1} \underline{u}_{1}^{(k-1)}+f_{1}\left(\underline{u}_{1}^{(k-1)}, \underline{u}_{2}^{(k-1)}\right), \\
& \left(\underline{u}_{2}^{(k)}\right)_{t}-L_{2} \underline{u}_{2}^{(k)}+M_{2} \bar{u}_{2}^{(k)}=M_{2} \underline{u}_{2}^{(k-1)}+f_{2}\left(\underline{u}_{1}^{(k-1)}, \underline{u}_{2}^{(k-1)}, \bar{u}_{3}^{(k-1)}\right), \\
& \left(\underline{u}_{3}^{(k)}\right)_{t}-L_{3} \underline{u}_{3}^{(k)}+M_{3} \underline{u}_{3}^{(k)}=M_{3} \underline{u}_{3}^{(k-1)}+f_{3}\left(\bar{u}_{1}^{(k-1)}, \underline{u}_{2}^{(k-1)}, \underline{u}_{3}^{(k-1)}\right)
\end{aligned}
$$

for $(x, t) \in Q_{T}$, and

$$
\begin{aligned}
B_{i}\left[\bar{u}_{i}^{(k)}\right] & =h_{i}(x)=B_{i}\left[\underline{u}_{i}^{(k)}\right], \quad i=1,2,3, \quad(x, t) \in S_{T}, \\
\bar{u}_{i}^{(k)}(x, 0) & =u_{i 0}(x)=\underline{u}_{i}^{(k)}(x, 0), \quad i=1,2,3, \quad x \in \Omega .
\end{aligned}
$$

By using standard techniques (e.g., see [12]), we can establish the following existence-comparison theorem. 


\section{THEOREM 2.1}

Suppose that the assumptions (2.4) hold and that there exists a pair of upper and lower solutions $\bar{U}=\left(\bar{u}_{1}, \bar{u}_{2}, \bar{u}_{3}\right)$ and $\underline{U}=\left(\underline{u}_{1}, \underline{u}_{2}, \underline{u}_{3}\right)$ satisfying $\underline{u}_{i} \leqslant \bar{u}_{i}$, $i=1,2,3$. Then the sequences $\left\{\bar{U}^{(k)}\right\}$ and $\left\{\underline{U}^{(k)}\right\}$ obtained as above converge monotonically from above and below, respectively, to a unique solution $U=$ $\left(u_{1}, u_{2}, u_{3}\right)$ of $(2.1)-(2.3)$ such that

$$
\underline{u}_{i}(x, t) \leqslant u_{i}(x, t) \leqslant \bar{u}_{i}(x, t), \quad i=1,2,3, \quad(x, t) \in Q_{T} .
$$

\section{NEUMANN PROBLEM}

In this section we study the homogeneous Neumann problem (1.2)-(1.4).

In view of Theorem 2.1, to obtain the existence and uniqueness of solutions of (1.2)-(1.4), we need only find a pair of upper and lower solutions of (1.2)-(1.4). We do it as follows. Consider the O.D.E. problem

$$
\begin{aligned}
& u_{1}^{\prime}(t)=\gamma u_{1}(t)\left(1-\frac{u_{1}(t)}{L_{0}+l M}\right), \\
& u_{2}^{\prime}(t)=\alpha u_{2}(t)\left(1-\frac{u_{2}(t)}{K}\right), \\
& u_{3}^{\prime}(t)=u_{3}(t)\left[-s+c \beta u_{2}(t)\right], \\
& u_{i}(0)=\hat{u}_{i} \equiv \sup _{\Omega} u_{i 0}(x)>0, \quad i=1,2,3
\end{aligned}
$$

where $M$ is a positive constant which will be chosen after we solve the second equation of (3.1). We have first

$$
u_{2}(t)=K\left[1+\frac{K-\hat{u}_{2}}{\hat{u}_{2}} e^{-\alpha t}\right]^{-1}
$$

Then, taking $M=\sup _{t \geqslant 0} u_{2}(t)=\max \left(K, \hat{u}_{2}\right)$, we have

$$
u_{1}(t)=\left[\frac{1}{L_{0}+l M}+\left(\frac{1}{\hat{u}_{1}}-\frac{1}{L_{0}+l M}\right) e^{-\gamma t}\right]^{-1}
$$

Thus

$$
u_{3}(t)=\hat{u}_{3} \exp \left(\int_{0}^{t}\left[-s+c \beta u_{2}(\tau)\right] d \tau\right)
$$

Clearly, $(0,0,0)$ and $\left(u_{1}(t), u_{2}(t), u_{3}(t)\right)$ are a pair of lower and upper solutions of (1.2)-(1.4). Hence we can use Theorem 2.1 for any $T>0$ and 
obtain

THEOREM 3.1

There exists a unique solution $\left(u_{1}(x, t), u_{2}(x, t), u_{3}(x, t)\right)$ satisfying

$$
0 \leqslant u_{i}(x, t) \leqslant u_{i}(t), \quad i=1,2,3 \text {. }
$$

where $u_{i}(t)$ is the solution of $(3.1), i=1,2,3$.

We have established the global existence of the solution of (1.2)-(1.4). Now, let us prove the global boundedness of the solution.

From (3.2) and (3.3), it is easy to see that

$$
\begin{aligned}
& u_{2}(x, t) \leqslant u_{2}(t) \leqslant \max \left(K, \hat{u}_{2}\right) \equiv M, \\
& u_{1}(x, t) \leqslant u_{1}(t) \leqslant \max \left(\hat{u}_{1}, L_{0}+l M\right) .
\end{aligned}
$$

Hence, it remains to prove the global boundedness of $u_{3}(x, t)$. Suppose that $\Omega$ is a bounded smooth domain where the Nirenberg-Gagliardo inequalities [16] and the divergence theorem hold, and that $u_{i 0}(x) \in C^{2}(\Omega) \cap L^{\infty}(\Omega)$, $i=1,2,3$. With these assumptions we have the following two lemmas, which will be proved in the Appendix.

\section{LEMMA 3.1}

Let $U(x, t)=\left(u_{1}(x, t), u_{2}(x, t), u_{3}(x, t)\right)$ be the solution of $(1.2)-(1.4)$. If there exists $p, 1 \leqslant p<\infty$, such that $\left\|u_{3}\right\|_{L^{p}(\Omega)}$ is uniformly bounded for $t \geqslant 0$, then $\left\|u_{3}\right\|_{L^{4}(\Omega)}$ is uniformly bounded for $t \geqslant 0$ and $q=p \cdot 2^{N}, N=1,2, \ldots$.

LEMMA 3.2

Let $U(x, t)=\left(u_{1}(x, t), u_{2}(x, t), u_{3}(x, t)\right)$ be the solution of (1.2)-(1.4). Then $\left\|u_{3}\right\|_{L^{1}(\Omega)}$ is uniformly bounded for $t \geqslant 0$.

Thus, by induction, we have

\section{THEOREM 3.2}

The solution $U(x, t)=\left(u_{1}(x, t), u_{2}(x, t), u_{3}(x, t)\right)$ of $(1.2)-(1.4)$ is uniformly bounded for $(x, t) \in \Omega \times \mathbb{R}^{+}$. In particular,

$$
\begin{aligned}
& u_{2}(x, t) \leqslant \max \left(K, \hat{u}_{2}\right), \\
& u_{1}(x, t) \leqslant \max \left(\hat{u}_{1}, L_{0}+l M\right),
\end{aligned}
$$

where

$$
\hat{u}_{i}=\sup _{\Omega} u_{i 0}(x), \quad i=1,2, \quad M=\max \left(K, \hat{u}_{2}\right) .
$$

Next, we analyze the asymptotic behavior of the three populations. We first give a sufficient condition for the extinction of the predator population $u_{3}$. 


\section{THEOREM 3.3}

Let $U(x, t)=\left(u_{1}(x, t), u_{2}(x, t), u_{3}(x, t)\right)$ be the solution of $(1.2)-(1.4)$. Suppose $-s+c \beta K /\left(1+m L_{0}\right)<0$. Then

$$
\lim _{t \rightarrow \infty}\left(u_{1}(x, t), u_{2}(x, t), u_{3}(x, t)\right)=\left(L_{0}+I K, K, 0\right) .
$$

Proof. We construct the desired lower and upper solutions of (1.2)-(1.4) as follows. Consider an O.D.E. system

$$
\begin{aligned}
& \bar{u}_{1}^{\prime}(t)=\gamma \bar{u}_{1}(t)\left(1-\frac{\bar{u}_{1}(t)}{L_{0}+l \bar{u}_{2}(t)}\right), \\
& \bar{u}_{2}^{\prime}(t)=\alpha \bar{u}_{2}(t)\left(1-\frac{\bar{u}_{2}(t)}{K}\right), \\
& \bar{u}_{3}^{\prime}(t)=\bar{u}_{3}(t)\left(-s+\frac{c \beta \bar{u}_{2}(t)}{1+m \underline{u}_{1}(t)}\right), \\
& \underline{u}_{1}^{\prime}(t)=\gamma \underline{u}_{1}(t)\left(1-\frac{\underline{u}_{1}(t)}{L_{0}+l \underline{u}_{2}(t)}\right), \\
& \underline{u}_{2}^{\prime}(t)=\alpha \underline{u}_{2}(t)\left(1-\frac{\underline{u}_{2}(t)}{K}\right)-\frac{\beta \underline{u}_{2}(t) \bar{u}_{3}(t)}{1+m \underline{u}_{1}(t),} \\
& \bar{u}_{i}(0)=\hat{u}_{i} \equiv \sup _{\Omega} u_{i 0}(x)>0, \quad i=1,2,3, \\
& \underline{u}_{i}(0)=\breve{u}_{i} \equiv \inf _{\Omega} u_{i 0}(x)>0, \quad i=1,2 .
\end{aligned}
$$

Obviously, $\left(\underline{u}_{1}(t), \underline{u}_{2}(t), 0\right)$ and $\left(\bar{u}_{1}(t), \bar{u}_{2}(t), \bar{u}_{3}(t)\right)$ are a pair of lower and upper solutions of (1.2)-(1.4). Moreover

$$
0 \leqslant \underline{u}_{2}(t) \leqslant \bar{u}_{2}(t)=K\left[1+\frac{K-\hat{u}_{2}}{\hat{u}_{2}} e^{-\alpha t}\right]^{-1}
$$

and

$$
\begin{aligned}
\underline{u}_{1}(t) & =\left[\frac{1}{\check{u}_{1}} e^{-\gamma t}+\int_{0}^{t} \frac{\gamma}{L_{0}+\underline{u}_{2}(\tau)} e^{-\gamma(t-\tau)} d \tau\right]^{-1} \\
& \geqslant\left[\frac{1}{\check{u}} e^{-\gamma t}+\int_{0}^{t} \frac{\gamma}{L_{0}} e^{-\gamma(t-\tau)} d \tau\right]^{-1}
\end{aligned}
$$

Therefore

$$
\liminf _{t \rightarrow \infty} \underline{u}_{1}(t) \geqslant \lim _{t \rightarrow \infty}\left[\frac{1}{\check{u}_{1}} e^{-\gamma t}+\int_{0}^{t} \frac{\gamma}{L_{0}} e^{-\gamma(t-\tau)} d \tau\right]^{-1}=L_{0}
$$


Set

$$
\varepsilon=s-\frac{c \beta K}{1+L_{0} m} .
$$

Then there exists $T_{0}>0$ such that

$$
-s+\frac{c \beta \bar{u}_{2}(t)}{1+m \underline{u}_{1}(t)}<-\frac{\varepsilon}{2} \quad \text { for } \quad t>T_{0} .
$$

Thus

$\bar{u}_{3}(t)=\hat{u}_{3} \exp \left\{\int_{0}^{T_{0}}\left(-s+\frac{c \beta \bar{u}_{2}(\tau)}{1+m \underline{u}_{1}(\tau)}\right) d \tau+\int_{T_{0}}^{t}\left(-s+\frac{c \beta \bar{u}_{2}(\tau)}{1+m \underline{u}_{1}(\tau)}\right) d \tau\right\}$,

and hence

$$
\lim _{t \rightarrow \infty} u_{3}(x, t) \leqslant \lim _{t \rightarrow \infty} \bar{u}_{3}(t) \leqslant \lim _{t \rightarrow \infty} \hat{u}_{3} \exp \left\{\text { constant }-\frac{\varepsilon}{2}\left(t-T_{0}\right)\right\}=0
$$

uniformly for $x \in \Omega$.

Now, let us determine the asymptotic behavior of the mutualist population $u_{1}(x, t)$ and prey population $u_{2}(x, t)$. Compare

$$
\begin{aligned}
& \underline{u}_{1}^{\prime}(t)=\gamma \underline{u}_{1}(t)\left(1-\frac{\underline{u}_{1}(t)}{L_{0}+\underline{u}_{2}(t)}\right), \\
& \underline{u}_{2}^{\prime}(t)=\alpha \underline{u}_{2}(t)\left(1-\frac{\underline{u}_{2}(t)}{K}\right)-\frac{\beta \underline{u}_{2}(t) \bar{u}_{3}(t)}{1+m u_{1}(t)}, \\
& \underline{u}_{i}(0)=\check{u}_{i}=\inf _{\Omega} u_{i 0}(x)>0, \quad i=1,2,
\end{aligned}
$$

with initial problem

$$
\begin{aligned}
& v_{2}^{\prime}(t)=\alpha v_{2}(t)\left(1-\frac{v_{2}(t)}{K}\right), \\
& v_{2}(0)=\breve{u}_{2} .
\end{aligned}
$$

Note that

$$
\sup _{t \geqslant 0}\left|\underline{u}_{2}(t)\right| \leqslant M<\infty
$$

and

$$
\lim _{t \rightarrow \infty} \bar{u}_{3}(t)=0
$$


By using a theorem of Markus [10], we have

$$
\lim _{t \rightarrow \infty}\left|\underline{u}_{2}(t)-v_{2}(t)\right|=0
$$

On the other hand,

$$
\lim _{t \rightarrow \infty} v_{2}(t)=\lim _{t \rightarrow \infty} K\left[1+\frac{K-\check{u}_{2}}{\check{u}_{2}} e^{-\alpha t}\right]^{-1}=K .
$$

Thus

$$
\lim _{t \rightarrow \infty} \underline{u}_{2}(t)=K
$$

and hence

$$
\begin{aligned}
\lim _{t \rightarrow \infty} \underline{u}_{1}(t) & =\lim _{t \rightarrow \infty}\left[\frac{1}{\breve{u}_{1}} e^{-\gamma t}+\int_{0}^{t} \frac{\gamma}{L_{0}+l \underline{u}_{2}(t)} e^{-\gamma(t-\tau)} d \tau\right]^{-1} \\
& =L_{0}+l K
\end{aligned}
$$

The fact that

$$
\lim _{t \rightarrow \infty} \bar{u}_{2}(t)=K \text { and } \quad \lim _{t \rightarrow \infty} \bar{u}_{1}(t)=L_{0}+l K
$$

is obvious.

The proof of the theorem is complete.

We see that under conditions of Theorem 3.3, the predator population $u_{3}$ goes to extinction, while populations $u_{1}$ and $u_{2}$ tend to constant states $L_{0}+I K$ and $K$, respectively.

We furthermore consider the all possible constant equilibrium solutions of (1.2)-(1.4), i.e., the solutions of

$$
\begin{aligned}
\gamma u_{1}\left(1-\frac{u_{1}}{L_{0}+l u_{2}}\right) & =0, \\
\alpha u_{2}\left(1-\frac{u_{2}}{K}\right)-\frac{\beta u_{2} u_{3}}{1+m u_{1}} & =0, \\
u_{3}\left(-s+\frac{c \beta u_{2}}{1+m u_{1}}\right) & =0
\end{aligned}
$$


All possible solutions of (3.7) [13] are given by

$E_{0}=(0,0,0), \quad E_{1}=(0, K, 0), \quad E_{2}=\left(0, \frac{s}{c \beta}, \frac{\alpha}{\beta}\left(1-\frac{s}{c \beta K}\right)\right), \quad E_{3}=\left(L_{0}, 0,0\right)$,

$E_{4}=\left(L_{0}+l K, K, 0\right), \quad E_{5}=\left(L_{0}+\frac{l s \lambda}{c \beta}, \frac{s \lambda}{c \beta}, \frac{\alpha \lambda}{\beta}\left(1-\frac{s \lambda}{c \beta K}\right)\right)$,

where $\lambda=c \beta\left(1+m L_{0}\right) /(c \beta-l m s)$.

In order to analyze the stability of these equilibria, ve rewrite (1.2) in the form in the Banach space $X=\oplus_{1}^{3} C^{2}(\Omega) \cap L^{3}(\Omega)$;

$$
\frac{d U}{d t}=A U+F(U)
$$

where

$$
\begin{aligned}
A & =\left[\begin{array}{ccc}
d_{1} \Delta+\gamma & & \\
& d_{2} \Delta+\alpha & \\
& d_{3} \Delta-s
\end{array}\right]=\left[\begin{array}{lll}
A_{1} & \\
& A_{2} & \\
& A_{3}
\end{array}\right], \\
F(U) & =\left(-\frac{\gamma u_{1}^{2}}{L_{0}+l u_{2}},-\frac{\alpha}{K} u_{2}^{2}-\frac{\beta u_{2} u_{3}}{1+m u_{1}}, \frac{c \beta u_{2} u_{3}}{1+m u_{1}}\right)^{T}, \\
D(A) & =\left\{U \in X:\left.\frac{\partial U}{\partial n}\right|_{\partial \Omega}=0\right\}
\end{aligned}
$$

The linearizations of the right side of (3.8) about $E_{i}, i=0,1, \ldots, 5$, respectively, are

$$
\begin{aligned}
& M_{0}\left(E_{0}\right)=\left[\begin{array}{lll}
d_{1} \Delta+\gamma & & \\
& d_{2} \Delta+\alpha & \\
& & d_{3} \Delta-s
\end{array}\right], \\
& M_{1}(E)=\left[\begin{array}{ccc}
d_{1} \Delta+\gamma & & \\
& d_{2} \Delta-\alpha & -\beta K \\
& & d_{3} \Delta-s+c \beta K
\end{array}\right] \text {, } \\
& M_{2}\left(E_{2}\right)=\left[\begin{array}{ccc}
d_{1} \Delta+\gamma & 0 & 0 \\
\frac{m s \alpha}{c \beta}\left(1-\frac{s}{c \beta K}\right) & d_{2} \Delta-\frac{\alpha s}{c \beta K} & -\frac{s}{c} \\
-m s \frac{\alpha}{\beta}\left(1-\frac{s \lambda}{c \beta K}\right) & c \alpha\left(1-\frac{s}{c \beta K}\right) & d_{3} \Delta
\end{array}\right] \text {, }
\end{aligned}
$$




$$
\begin{aligned}
M_{3}\left(E_{3}\right)= & {\left[\begin{array}{ccc}
d_{1} \Delta-\gamma & \gamma l & \\
& d_{2} \Delta+d & \\
M_{4}\left(E_{4}\right)= & d_{3} \Delta-s
\end{array}\right], } \\
& {\left[\begin{array}{ccc}
d_{1} \Delta-\gamma & \gamma l & 0 \\
0 & d_{2} \Delta-d & -\frac{\beta K}{1+m\left(L_{0}+l K\right)} \\
0 & 0 & d_{3} \Delta-s+\frac{c \beta K}{1+m\left(L_{0}+l K\right)}
\end{array}\right], } \\
M_{5}\left(E_{5}\right)= & \left.\begin{array}{ccc}
d_{1} \Delta-\gamma & \\
\frac{m s d}{c \beta}\left(1-\frac{s \lambda}{c \beta K}\right) & d_{2} \Delta-\frac{d s \lambda}{c \beta K} & -\frac{s}{c} \\
-m s \frac{\alpha}{\beta}\left(1-\frac{s \lambda}{c \beta K}\right) & c \alpha\left(1-\frac{s \lambda}{c \beta K}\right) & d_{3} \Delta
\end{array}\right] .
\end{aligned}
$$

Denote

$$
\begin{aligned}
P_{i}(\eta, \Delta) & =\operatorname{det}\left(\eta I-M_{i}\right) \\
\Lambda_{i} & =\left\{\eta: P_{i}(\eta, \mu)=0 \text { for some } \mu \in \sigma(\Delta)\right\}
\end{aligned}
$$

It is easy to show that $[6,8]$

$$
\sigma\left(M_{i}\right) \subset \Lambda_{i}, \quad i=0,1, \ldots, 5,
$$

where, e.g., $\sigma(\Delta)$ denotes the point spectrum of $\Delta$ with homogeneous Neumann boundary condition. It is well known that $\sigma(\Delta)$ is an infinite but discrete set of simple real eigenvalues bounded from above, namely

$$
0=\mu_{0}>\mu_{1}>\cdots>\mu_{n}>\cdots
$$

Obviously, none of $E_{0}, E_{1}, E_{2}$, and $E_{3}$ is stable, since the corresponding $P_{i}(\eta, \mu)(i=0,1,2,3)$ has at least one positive root for $\mu_{0}=0 \in \sigma(\Delta)$. Since $d_{i} \mu \leqslant 0$ for $\mu \in \sigma(A), i=1,2,3, E_{4}$ is linearly stable [15] if

$$
s>\frac{c \beta K}{1+m\left(L_{0}+I K\right)} .
$$

As shown in [13], $E_{5}$ exists if

$$
s<\frac{c \beta K}{1+m\left(L_{0}+l K\right)}
$$


Let us analyze the spectrum of $M_{5}\left(E_{5}\right)$. Set

$$
P_{5}=\eta^{3}+a_{1} \eta^{2}+a_{2} \eta+a_{3} \quad \text { for } \quad \mu \in \sigma(A) \text {. }
$$

Then

$$
\begin{aligned}
a_{1}= & -\mu\left(d_{1}+d_{2}+d_{3}\right)+\gamma+\frac{\alpha s \lambda}{c \beta K}>0 \\
a_{2}= & \mu^{2}\left(d_{1} d_{2}+d_{1} d_{3}+d_{2} d_{3}\right)-\mu \gamma\left(d_{2}+d_{3}\right)-\mu \frac{\alpha s \lambda}{c \beta K}\left(d_{1}+d_{3}\right) \\
& +\frac{\alpha s \lambda}{c \beta K} \gamma+\left(1-\frac{s \lambda}{c \beta K}\right)\left(1-\frac{\gamma l m}{c \beta}\right) \alpha s \\
a_{3}= & -\mu^{3} d_{1} d_{2} d_{3}+\mu^{2} \gamma d_{2} d_{3}+\mu^{2} \frac{\alpha s \lambda}{c \beta K} d_{1} d_{3}-\mu \frac{\alpha s \lambda}{c \beta K} \gamma d_{3} \\
& +\frac{\alpha s}{c \beta}\left(1-\frac{s \lambda}{c \beta K}\right)\left[-\mu d_{1} c \beta+\mu d_{3} \operatorname{lm} \gamma+\gamma(c \beta-\operatorname{lms})\right]
\end{aligned}
$$

Note that (3.12) implies $1-s \lambda / c \beta K>0$ and $c \beta>l m s$. So $a_{3}>0$ if

$$
c \beta d_{1}-\left[\operatorname{lm}-\frac{\lambda}{K}\left(1-\frac{s \lambda}{c \beta K}\right)^{-1}\right] \gamma d_{3} \geqslant 0 .
$$

Through a tedious calculation, we get that $a_{1} a_{2}-a_{3}>0$ if

$$
s \geqslant \gamma
$$

and

$$
\begin{aligned}
\frac{\alpha s}{c \beta}\left(1-\frac{s \lambda}{c \beta K}\right)\left(d_{3} c \beta-d_{1} \gamma / m\right) & +2 \frac{\alpha s \lambda \gamma}{c \beta K}\left(d_{1}+d_{2}+d_{3}\right) \\
& +\gamma^{2}\left(d_{2}+d_{3}\right)+\left(\frac{\alpha s \lambda}{c \beta K}\right)^{2}\left(d_{1}+d_{3}\right) \geqslant 0 .
\end{aligned}
$$

By the Routh-Hurwitz criteria, $E_{5}$ is linearly stable if (3.12)-(3.15) hold.

We have proved

\section{TIIEOREM 3.4}

(i) None of $E_{0}, E_{1}, E_{2}$, and $E_{3}$ are stable.

(ii) $E_{4}$ is linearly stable if (3.11) holds.

(iii) $E_{5}$ is linearly stable if (3.12)-(3.15) hold. 
In the final part of this section, we study the global asymptotic stability of $E_{4}$ and $E_{5}$. Rewrite (1.2)-(1.4) as follows:

$$
\begin{aligned}
\frac{\partial U}{\partial t} & =D \Delta U+f(U) & & \text { in } \Omega \times \mathbb{R}^{+}, \\
\frac{\partial U}{\partial n} & =0 & & \text { on } \partial \Omega \times \mathbb{R}^{+}, \\
U(x, 0) & =U_{0}(x)>0, & & \text { on } \Omega,
\end{aligned}
$$

where

$$
\begin{array}{r}
D=\left[\begin{array}{lll}
d_{1} & & \\
& d_{2} & \\
& & d_{3}
\end{array}\right], \quad U=\left[\begin{array}{l}
u_{1}(x, t) \\
u_{2}(x, t) \\
u_{3}(x, t)
\end{array}\right], \\
f(U)=\left[\gamma u_{1}\left(1-\frac{u_{1}}{I_{0}+l u_{2}}\right), \alpha u_{2}\left(1-\frac{u_{2}}{K}\right)-\frac{\beta u_{2} u_{3}}{1+m u_{1}},\right. \\
\left.u_{3}\left(-s+\frac{c \beta u_{2}}{1+m u_{1}}\right)\right]^{T} .
\end{array}
$$

From Theorem 3.2, it has been shown that the solution has a positively invariant region [2] $\Sigma \subset \mathbb{R}^{n}$, where $\Sigma$ is a compact set. Let $\mu \equiv-\mu_{1}>0$ be the smallest nonzero eigenvalue of $-\Delta$ with homogeneous Neumann boundary condition,

$$
d=\min \left(d_{1}, d_{2}, d_{3}\right), \quad M=\max \left\{\left|d f_{u}\right|: U \in \Sigma\right\}, \quad \sigma=\mu d-M
$$

THEORLM 3.5 (L. CONWAY, D. HOFF AND J. SMOLLER [3])

Assume $\sigma>0$, and $U$ is a solution of (3.16) with $U_{0} \in K(K \subset \mathbb{R}$ " is a compact set). Then there exist constants $C_{i}, i=1,2$ such that

$$
\|U(x, t)-\bar{U}(t)\|_{1, x} \leqslant C_{1} e^{-\sigma t},
$$

where

$$
\bar{U}(t)=\frac{1}{\operatorname{mes}(\Omega)} \int_{\Omega} U(x, t) d x
$$


satisfying

$$
\begin{aligned}
\frac{d \bar{U}(t)}{d t} & =f(\bar{U})+g(t), \\
\bar{U}(0) & =\frac{1}{\operatorname{mes}(\Omega)} \int_{\Omega} U_{0}(x) d x,
\end{aligned}
$$

and

$$
|g(t)| \leqslant C_{2} e^{-\sigma t}
$$

Now compare $\bar{U}(t)$ with the solution $V(t)$ of

$$
\begin{aligned}
\frac{d V}{d t} & =f(V), \\
V(0) & =\frac{1}{\operatorname{mes}(\Omega)} \int_{\Omega} U_{0}(x) d x
\end{aligned}
$$

By Markus's theorem, it can be shown that

$$
\lim _{t \rightarrow \infty}|V(t)-\bar{U}(t)|=0 .
$$

Combining the above results, we have

THEOREM 3.6

Suppose $\sigma>0, U_{0}(x) \in \Sigma$. Then

(i) $\lim _{t \rightarrow \infty} U(x, t)=\left(L_{0}+I K, K, 0\right)$ if (3.11) holds.

(ii) $\lim _{t \rightarrow \infty} U(x, t)=\left(L_{0}+l s \lambda / c \beta, s \lambda / c \beta,(\alpha \lambda / \beta)(1-s \lambda / c \beta K)\right)$ if (3.12)-(3.15) hold.

\section{DIRICHLET PROBLEM}

In this section we discuss Dirichlet the problem (1.2), (1.3), (1.5). First, let us construct a pair of lower and upper solutions to establish the existence and uniqueness of solution of this problem.

Let $\lambda_{0}$ be the principal eigenvalue of $-\Delta$ with homogeneous Dirichlet boundary condition. Then we can construct a function $\tilde{\varphi}_{0}(x)[5,14]$, normalized by $\sup _{x \in \Omega} \tilde{\varphi}_{0}(x)=1$, such that

$$
\begin{aligned}
\Delta \tilde{\varphi}_{0}+\lambda_{0} \tilde{\varphi}_{0} \leqslant 0, & x \in \Omega, \\
\tilde{\varphi}_{0}>0, & x \in \bar{\Omega} .
\end{aligned}
$$

Therefore, there exist constants $M_{i}, i=1,2,3$, such that

$$
u_{i 0}(x) \leqslant M_{i} \tilde{\varphi}_{0}(x), \quad i=1,2,3, \quad x \in \bar{\Omega} .
$$


Set

$$
\begin{aligned}
& \bar{u}_{1}(x, t)=M_{1} \tilde{\varphi}_{0}(x) \exp \left\{\left(-d_{1} \lambda_{0}+\gamma\right) t\right\}, \\
& \bar{u}_{2}(x, t)=M_{2} \tilde{\varphi}_{0}(x) \exp \left\{\left(-d_{2} \lambda_{0}+\alpha\right) t\right\}, \quad(x, t) \in \Omega \times \mathbb{R}^{+} \\
& \bar{u}_{3}(x, t)=M_{3} \tilde{\varphi}_{0}(x) \exp \left\{\left(-d_{3} \lambda_{0}-s+c \beta K_{1}\right) t\right\},
\end{aligned}
$$

where $K_{1}=\max \left\{K, M_{2}\right\}$.

It is easy to check that, $(0,0,0)$ and $\left(\bar{u}_{1}, \bar{u}_{2}, \bar{u}_{3}\right)$ define a pair of lower and upper solutions of (1.2), (1.3), and (1.5). In fact, if we rewrite (1.2) in the form

$$
\begin{aligned}
& \frac{\partial u_{1}}{\partial t}-d_{1} \Delta u_{1}=f_{1}\left(u_{1}, u_{2}\right), \\
& \frac{\partial u_{2}}{\partial t}-d_{2} \Delta u_{2}=f_{2}\left(u_{1}, u_{2}, u_{3}\right),(x, t) \in \Omega \times \mathbb{R}^{+}, \\
& \frac{\partial u_{3}}{\partial t}-d_{3} \Delta u_{3}=f_{3}\left(u_{1}, u_{2}, u_{3}\right),
\end{aligned}
$$

then, in view of (4.1), we have

$$
\begin{aligned}
\frac{\partial \bar{u}_{3}}{\partial t} & =\left(-d_{3} \lambda_{0}-s+c \beta K_{1}\right) \bar{u}_{3} \\
& \geqslant d_{3} \Delta \bar{u}_{3}+\left(-s+c \beta K_{1}\right) \bar{u}_{3} \\
& \geqslant d_{3} \Delta \bar{u}_{3}+\left(-s+c \beta \bar{u}_{2}\right) \bar{u}_{3} \\
& =d_{3} \Delta \bar{u}_{3}+f_{3}\left(\underline{u}_{1}, \bar{u}_{2}, \bar{u}_{3}\right), \quad(x, t) \in \Omega \times \mathbb{R}^{+} .
\end{aligned}
$$

Similarly, it can be shown that

$$
\begin{aligned}
& \frac{\partial \bar{u}_{1}}{\partial t}-d_{1} \Delta \bar{u}_{1} \geqslant f_{1}\left(\bar{u}_{1}, \bar{u}_{2}\right), \quad(x, t) \in \Omega \times \mathbb{R}^{+}, \\
& \frac{\partial \bar{u}_{2}}{\partial t}-d_{2} \Delta \bar{u}_{2} \geqslant f_{2}\left(\bar{u}_{1}, \bar{u}_{2}, \underline{u}_{3}\right),(x, t) \in \Omega \times \mathbb{R}^{+} .
\end{aligned}
$$

In addition, clearly, the following inequalities hold:

$$
\begin{aligned}
& \bar{u}_{i}(x, 0) \geqslant u_{i 0}(x), \quad i=1,2,3, \quad x \in \bar{\Omega}, \\
& \left.\bar{u}_{i}(x, t)\right|_{\lambda \Omega} \geqslant 0, \quad i=1,2,3 \text {. }
\end{aligned}
$$

The inequalities (4.5)-(4.9) mean that $\left(\bar{u}, \bar{u}_{2}, \bar{u}_{3}\right)$ is an upper solution of (1.2), (1.3), and (1.5) with $(0,0,0)$ as the corresponding lower solution. So, by using Theorem 2.1, we get immediately the following theorem: 


\section{THEOREM 4.1}

There exists the unique solution $\left(u_{1}(x, t), u_{2}(x, t), u_{3}(x, t)\right)$ of the problem (1.2), (1.3), (1.5) satisfying

$$
0 \leqslant u_{i}(x, t) \leqslant \bar{u}_{i}(x, t), \quad i=1,2,3, \quad(x, t) \in \Omega \times \mathbb{P}^{+},
$$

where $\bar{u}_{i}(x, t)(i=1,2,3)$ is defined by $(4.3)$.

In view of the ecological origin of the model, we are interested in the nonnegative solutions only. In fact, it is very easy to check that (1.2) has an invariant region $\left\{\left(u_{1}, u_{2}, u_{3}\right) \in \mathbb{R}^{3}, u_{i} \geqslant 0, i=1,2,3\right\}$. Now, we can show that the solution is bounded from above as well.

Let $T_{0}<\infty$ be an arbitrary constant. Suppose $u_{2}(x, t)$ attains its maximum at $\left(x_{0}, t_{0}\right) \in \Omega \times\left(0, T_{0}\right]$. Then we have

$$
\frac{\partial u_{2}\left(x_{0}, t_{0}\right)}{\partial t} \geqslant 0 \text { and } \Delta u_{2}\left(x_{0}, t_{0}\right) \leqslant 0
$$

In view of (4.10), the system (1.2) together with the nonnegativity of the solution implies

$$
0 \leqslant u_{2}(x, t) \leqslant K, \quad(x, t) \in \Omega \times\left(0, T_{0}\right] .
$$

By the arbitrariness of $T_{0}$, we deduce

$$
0 \leqslant u_{2}(x, t) \leqslant K, \quad(x, t) \in \Omega \times \mathbb{R}^{+} .
$$

In the same way, we can prove

$$
0 \leqslant u_{1}(x, t) \leqslant L_{0}+l K, \quad(x, t) \in \Omega \times \mathbb{R}^{+} .
$$

Notice that (1.5) and the nonnegativity of the solution imply $\partial u_{i} /\left.\partial n\right|_{\lambda \Omega}$ $\leqslant 0, i=1,2,3$. So we can use Lemmas 3.1 and 3.2 to prove that

$$
0 \leqslant u_{3}(x, t) \leqslant \text { constant }<\infty, \quad(x, t) \in \Omega \times \mathbb{R}^{+} .
$$

Thus, we get the following theorem concerning the boundedness of solution. THEOREM 4.2

Let $\left(u_{1}(x, t), u_{2}(x, t), u_{3}(x, t)\right)$ be the solution of (1.2), (1.3), and (1.5). Then

$$
\begin{aligned}
& 0 \leqslant u_{1}(x, t) \leqslant \max \left(L_{0}+I K, \hat{u}_{1}\right), \\
& 0 \leqslant u_{2}(x, t) \leqslant \max \left(K, \hat{u}_{2}\right), \quad(x, t) \in \Omega \times \mathbb{R}^{+}, \\
& 0 \leqslant u_{3}(x, t) \leqslant \hat{M}<\infty
\end{aligned}
$$

where $\hat{u}_{1}=\sup _{\Omega} u_{10}(x), \hat{u}_{2}=\sup _{\Omega} u_{20}(x)$, and $\hat{M}$ is a positive constant 
Next, we study the asymptotic behavior of the solution. To begin with, we discuss the asymptotic stability of the trivial equilibrium solution $(0,0,0)$.

THEOREM 4.3

Let $\lambda_{0}$ be the principal eigenvalue of $-\Delta$ with homogeneous Dirichlet boundary condition. Assume

$$
\Lambda-\max \left\{-d_{1} \lambda_{0}+\gamma,-d_{2} \lambda_{0}+\alpha\right\}<0
$$

Then $(0,0,0)$ is the only nonnegative equilibrium solution of (1.2), (1.3), and (1.5). Moreover, it is globally asymptotically stable.

Proof. Let $\left(u_{1}^{*}(x), u_{2}^{*}(x), u_{3}^{*}(x)\right)$ be an arbitrary nonnegative equilibrium solution, i.e.,

$$
\begin{aligned}
d_{1} \Delta u_{1}^{*}+\gamma u_{1}^{*}\left(1-\frac{u_{1}^{*}}{L_{0}+l u_{2}^{*}}\right) & =0, \\
d_{2} \Delta u_{2}^{*}+\alpha u_{2}^{*}\left(1-\frac{u_{2}^{*}}{K}\right)-\frac{\beta u_{2}^{*} u_{3}^{*}}{1+m u_{1}^{*}} & =0, \\
d_{3} \Delta u_{3}^{*}+u_{3}^{*}\left(-s+\frac{c \beta u_{2}^{*}}{1+m u_{1}^{*}}\right) & =0, \\
\left.u_{i}^{*}\right|_{\partial \Omega} & =0, \quad i=1,2,3 .
\end{aligned}
$$

This means that $u_{2}^{*}(x)$ is a nonnegative solution of the following linear problem:

$$
\begin{aligned}
d_{2} \Delta u+b(x) u & =0, \quad x \in \Omega, \\
\left.u\right|_{a \Omega} & =0,
\end{aligned}
$$

where $b(x)=\alpha-\alpha u_{2}^{*} / K-\beta u_{3}^{*} /\left(1+m u_{1}^{*}\right)$. Since $u_{i}^{*}(x) \geqslant 0$ for $i=1,2,3$ and $x \in \Omega$, it follows that

$$
b(x) \leqslant \alpha<d_{2} \lambda_{0}, \quad x \in \Omega .
$$

Therefore

$$
\left\{\frac{b(x)}{d_{2}}: x \in \Omega\right\} \cap \sigma(-\Delta)=\varnothing,
$$

where $\sigma(-\Delta)$ denotes the point spectrum of $-\Delta$ with homogeneous 
Dirichlet boundary condition. (4.13) implies

$$
\Delta u_{2}^{*}+\frac{b(x)}{d_{2}} u_{2}^{*} \neq 0 \quad \text { for any } \quad x \in \Omega
$$

whenever $u_{2}^{*}$ is not constant zero. Hence

$$
u_{2}^{*}(x) \equiv 0, \quad x \in \bar{\Omega} .
$$

It can be proved in the same way that

$$
u_{1}^{*}(x) \equiv 0, \quad x \in \bar{\Omega} .
$$

Due to (4.14) and (4.15), we know from (4.11) that $u_{3}^{*}(x)$ satisfies

$$
\begin{aligned}
d_{3} \Delta u_{3}^{*}-s u_{3}^{*} & =0, \quad x \in \Omega, \\
\left.u_{3}^{*}\right|_{\nexists \Omega} & =0 .
\end{aligned}
$$

By using the maximum principle, we have

$$
u_{3}^{*}(x) \equiv 0, \quad x \in \bar{\Omega} .
$$

Thus, $(0,0,0)$ is the unique nonnegative equilibrium solution of $(1.2),(1.3)$, and (1.5).

In order to prove its asymptotic stability, let us construct an upper solution with $(0,0,0)$ as corresponding lower solution. Set

$$
\begin{aligned}
& \tilde{u}_{1}(x, t)=M_{1} \tilde{\varphi}_{0} \exp \left\{\left(-d_{1} \lambda_{0}+\gamma\right) t\right\}, \\
& \tilde{u}_{2}(x, t)=M_{2} \tilde{\varphi}_{0} \exp \left\{\left(-d_{2} \lambda_{0}+\alpha\right) t\right\},(x, t) \in \Omega \times\left[T_{0}, \infty\right), \\
& \tilde{u}_{3}(x, t)=M_{3} \tilde{\varphi}_{0} \exp \left\{\left(-d_{3} \lambda_{0}-s+c \beta K_{2}\right) t\right\},
\end{aligned}
$$

where $\tilde{\varphi}_{0}$ and $M_{i}(i=1,2,3)$ are taken as in (4.1) and (4.2), $K_{2}=$ $M_{2} \exp \left\{-d_{2} \lambda_{0}+\alpha T_{0}\right\}$, and $T_{0}$ is sufficiently large so that

$$
c \beta K_{2}-d_{3} \lambda_{0}-s \leqslant-\frac{\delta}{2}<0 .
$$

We can check that $(0,0,0)$ and $\left(\tilde{u}_{1}, \tilde{u}_{2}, \tilde{u}_{3}\right)$ defined by $(4.18)$ consist of a pair of lower and upper solutions of (1.2), (1.3), and (1.5).

Since $\Lambda<0$, we have from (4.19) and (4.18) that

$$
\lim _{i \rightarrow \infty} \sup _{x \in \mathbb{S}} \tilde{u}_{i}=0, \quad i=1,2,3,
$$


and hence

$$
\lim _{t \rightarrow \infty} \sup _{x \in \Omega} u_{i}(x, t)=0, \quad i=1,2,3 \text {. }
$$

This completes the proof of Theorem 4.3.

If the habitat $\Omega$ is large enough (hence $\lambda_{0}$ is small enough), or the diffusion of $u_{1}$ or $u_{2}$ is weak enough, that $\Lambda>0$, then we will see that the problem (1.2), (1.3), (1.5) admits some nonconstant equilibrium solutions as well. In other words, bifurcations occur as $\Lambda$ passes through zero from negative to positive.

\section{THEOREM 4.4}

Assume $-d_{1} \lambda_{0}+\gamma>0$ and $-d_{2} \lambda_{0}+d<0$. Then there exists a nonconstant equilibrium solution $\left(u_{1}^{*}(x), 0,0\right)$ of $(1.2),(1.3)$, and (1.5). Moreover, it is asymptotically stable.

Proof. Consider the following Dirichlet problem for the semilinear elliptic equation:

$$
\begin{aligned}
d_{2} \Delta u_{1}+\gamma u_{1}\left(1-\frac{u_{1}}{L_{0}}\right) & =0, \quad x \in \Omega, \\
\left.u_{1}\right|_{\partial \Omega} & =0 .
\end{aligned}
$$

Set

$$
\begin{array}{ll}
\bar{u}_{1}(x)=M \tilde{\varphi}_{0}(x), & x \in \bar{\Omega}, \\
\underline{u}_{1}(x)=\varepsilon \varphi_{0}(x), & x \in \bar{\Omega},
\end{array}
$$

where $\tilde{\varphi}_{0}$ is taken as in (4.1); $\varphi_{0}$ is the principal eigenfunction of $-\Delta$ with homogeneous Dirichlet boundary condition; both of them are normalized by $\sup _{\Omega} \tilde{\varphi}_{0}=1$ and $\sup _{\Omega} \varphi_{0}=1$; and we have for the constants

$$
M \geqslant \frac{L_{0}\left(-d_{1} \lambda_{0}+\gamma\right)}{\gamma \min _{\bar{\Omega}} \tilde{\varphi}_{0}}, \quad \varepsilon \leqslant \frac{L_{0}\left(-d_{1} \lambda_{0}+\gamma\right)}{\gamma}
$$

It is easy to check that $\bar{u}_{1}(x)$ and $\underline{u}_{1}(x)$ defined by (4.22) and (4.23) are the upper and lower solutions in the sense of Theorem 10.3 of [15], respectively. By using that theorem of [15], we know that there exists a nonconstant solution $u_{1}^{*}(x)$ of (4.21) satisfying

$$
0<\underline{u}_{1}(x) \leqslant u_{1}^{*}(x) \leqslant \bar{u}_{1}(x), \quad x \in \Omega .
$$

This means that $U^{*}(*)=\left(u_{1}^{*}(x), 0,0\right)$ is a nonconstant equilibrium solution of (1.2), (1.3), and (1.5). 
To prove the stability of $U^{*}$, we linearize the reaction terms of (1.2) at $U^{*}$ and analyze the spectrum of the linearized operators. Rewrite (1.2) as an evolution equation in the Banach space $X=\oplus_{1}^{3} X_{i}=\oplus_{1}^{3} L^{2}(\Omega) \cap C^{2}(\Omega)$ as in Section 3:

$$
\frac{d U}{d t}=A U+F(U)
$$

where $A$ and $F(U)$ are as the same as (3.9) and (3.10), and

$$
D\left(A_{i}\right)=\left\{u_{i} \in X_{i},\left.u_{i}\right|_{\partial \Omega}=0, A_{i} u_{i} \in L^{2}(\Omega)\right\}, \quad i=1,2,3 .
$$

Linearizing $F(U)$ at $U^{*}=\left(u_{1}^{*}(x), 0,0\right)$, we get

$$
F\left(U+U^{*}\right)=F\left(U^{*}\right)+B U+g(U),
$$

where

$$
\begin{aligned}
g(U) & =o\left(\|U\|_{X}\right), \\
B & =\left[\begin{array}{ccc}
-\frac{2 \gamma u_{1}^{*}}{L_{0}} & \frac{l \gamma u_{1}^{* 2}}{L_{0}^{2}} & 0 \\
0 & 0 & 0 \\
0 & 0 & 0
\end{array}\right] .
\end{aligned}
$$

We need only analyze the spectrum of the operator $\mathscr{U}=A+B[6,7,11]$. The resolvent equation for $\mathscr{U}$ is

$$
(A+B-\mu I) U=V, \quad \mu \in \mathbb{C}, \quad V=\left(u_{1}, v_{2}, v_{3}\right)^{T} \in X,
$$

i.e.,

$$
\left[\begin{array}{ccc}
A_{1}-\frac{2 \gamma u_{1}^{*}}{L_{0}}-\mu & \frac{l \gamma u_{1}^{* 2}}{L_{0}^{2}} & 0 \\
0 & A_{2}-\mu & 0 \\
0 & 0 & A_{3}-\mu
\end{array}\right]\left[\begin{array}{l}
u_{1} \\
u_{2} \\
u_{3}
\end{array}\right]=\left[\begin{array}{l}
v_{1} \\
v_{2} \\
v_{3}
\end{array}\right]
$$

for $\mu \in \rho\left(A_{2}\right) \cap \rho\left(A_{3}\right)$, where $\rho\left(A_{i}\right)$ denotes the resolvent set of $A_{i}, i=2,3$. Hence

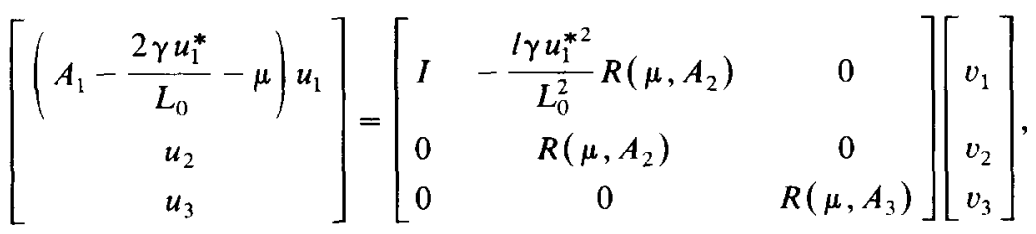


where $R\left(\mu, A_{2}\right)$ and $R\left(\mu, A_{3}\right)$ are the resolvent operators of $A_{2}$ and $A_{3}$, respectively. Set

$$
\begin{aligned}
\overline{A_{1}}(\mu) & =A_{1}-\frac{2 \gamma u_{1}^{*}}{L_{0}}-\mu, \\
\mathscr{R} & =\left\{\mu: \mu \in \rho\left(A_{2}\right) \cap \rho\left(A_{3}\right), \overline{A_{1}} \text { is invertible in } L^{2}(\Omega)\right\} .
\end{aligned}
$$

We have the following four lemmas:

I.EMMA 4.1

$$
\mathscr{R} \subset \rho(U)
$$

\section{I.I.MMA 4.2}

Let $\mu \in \rho\left(A_{2}\right) \cap \rho\left(A_{3}\right)$, then $\overline{A_{1}}(\mu)$ is invertible in $L^{2}(\Omega)$ if and only if zero is not an eigenvalue of $\overline{A_{1}}(\mu)$.

\section{ILISMA 4.3}

There exist $\theta^{*} \in(0, \pi / 2)$ and $\gamma^{*}>0$ such that

$$
S^{*} \equiv\left\{\mu \in \mathbb{C}:\left|\arg \left(\mu+\gamma^{*}\right)\right| \leqslant \frac{\pi}{2}+\theta^{*}\right\} \subset \mathscr{R} .
$$

IEMMA 4.4

$$
\sigma(\mathscr{U}) \subset\left\{\lambda \in \mathbb{C}: \operatorname{Re} \lambda<-\gamma^{*}\right\}
$$

Lemmas 3.1 and 3.2 are obvious [11]. Lemma 3.4 comes from Lemmas 3.1-3.3. The proof of Lemma 3.3 can be found in the Appendix.

We note that the linear stability of $U^{*}=\left(u_{1}^{*}, 0,0\right)$ is the straightforward result of Lemma 4.4. Thus, we complete the proof of the theorem. THEOREM 4.5

Assume $d_{1} \lambda_{0}+\gamma<0$ and $d_{2} \lambda_{0}+\alpha>0$. Then there exists a nonconstant equilibrium solution $\tilde{U}=\left(0, \tilde{u}_{2}(x), 0\right)$ of $(1.2),(1.3)$, and (1.5). Let

$$
K^{*}=\max \left\{\operatorname{Re} \lambda: \lambda \in \sigma\left(A_{2}-\frac{2 \alpha}{K} \tilde{u}_{2}\right) \cup \sigma\left(A_{3}+c \beta \tilde{u}_{2}\right)\right\}
$$

Then $\left(0, \tilde{u}_{2}(x), 0\right)$ is linearly stable if $K^{*}<0$ and is unstable if $K^{*}>0$.

Proof. We can prove the existence of the equilibrium solution $\left(0, \tilde{u}_{2}(x), 0\right)$ in the same way as we did in the proof of Theorem 4.4 . 
Linearizing $F(U)$ at $\tilde{U}$, we get

$$
\begin{gathered}
F(U+\tilde{U})=F(\tilde{U})+C U+g(U), \\
C=\left[\begin{array}{ccc}
0 & 0 & 0 \\
0 & -\frac{2 \alpha}{K} \tilde{u}_{2} & 0 \\
0 & 0 & c \beta \tilde{u}_{2}
\end{array}\right] .
\end{gathered}
$$

Set

$$
\mathscr{V}=A+C
$$

Then

$$
\mathscr{Y}-\mu I=\left[\begin{array}{ccc}
A_{1}-\mu & 0 & 0 \\
0 & A_{2}-\frac{2 \alpha}{K} \tilde{u}_{2}-\mu & 0 \\
0 & 0 & A_{3}+c \beta \tilde{u}_{2}-\mu
\end{array}\right]
$$

Clearly, the point spectrum of $\mathscr{V}$ is

$$
\sigma(\mathscr{V})=\sigma\left(A_{1}\right) \cup \sigma\left(A_{2}-\frac{2 \alpha}{K} \tilde{u}_{2}\right) \cup \sigma\left(A_{3}+c \beta \tilde{u}_{2}\right) .
$$

On the other hand, by the assumption we have

$$
\sigma\left(A_{1}\right) \subset\left\{\lambda \in \mathbb{C}: \operatorname{Re} \lambda \leqslant d_{1} \lambda_{0}+\gamma<0\right\} .
$$

The conclusions on the linear stability and on the instability come from (4.25) and (4.26).

The proof is complete.

\section{DISCUSSION}

We summarize and discuss our results as follows.

First, the solution exists uniquely and is bounded, regardless of the diffusion mechanism and the kinds of boundary value conditions. This is bccause of the biologically reasonable assumptions that the mutualist-prey $u_{2}$ has a finite carrying capacity and that the mutualist $u_{1}$ benefits $u_{2}$ only by deterring the predation on $u_{2}$.

Sccond, for the Neumann problem, as we see in Section 3, some results are somewhat similar to those of the original O.D.E. problem [13]. For example, for the extinction of the predator $u_{3}$, the Neumann problem and the O.D.E. problem have the same sufficient condition, i.e. (3.16) of [13]. This condition 
can be explained by the fact that the carrying capacity $K$ of the prey $u_{2}$ (with the given benefit of $u_{1}$ ) is too small relative to the death rate $s$ of the predator $u_{3}$. In addition, the constant equilibrium solutions $E_{0}, E_{1}, \ldots, E_{5}$ are the same for the Neumann problem and the O.D.E. problem. We know that the solutions of the O.D.E. problem can be considered independent of spatial variables and hence satisfy the homogeneous Neumann boundary condition automatically.

However, there are some evident differences between the Neumann problem and the O.D.E. problem. For example, as to the asymptotic stability of $E_{5}$, Theorem 3.4 says that in addition to $(3.12)$ and $(3.14)$ (i.e. (3.11) and (3.15) of [13]), we need also (3.13) and (3.15), which can be considered as the conditions that the diffusion constants have to satisfy.

We note that both Theorems 3.3 and 3.6 deal with global asymptotic stability, but they are different. For example, the conditions of Theorems 3.3 and 3.6(i) are different, and neither of them implies the other, although both concern the extinction of $u_{3}$. The inequality (3.11) of Theorem 3.6(i) is weaker than $-s+c \beta K /\left(1+m L_{0}\right)<0$ in Theorem 3.3, but Theorem 3.6(i) requires another condition $\sigma=\mu d-M>0$ as well, which can be written as $\mu>M / d$ or $d>M / \mu$. In the former case, one can view it as saying that $\mu$ is large and can interpret $\sigma>0$ as saying that the habitat $\Omega$ is small, since $\mu$ is inversely proportional to the squared diameter of $\Omega$ [4]. In the latter case, $\sigma>0$ can be looked upon as saying that the diffusion is strong relative to the reaction terms. So, with small $\Omega$ and large diffusion, it is reasonable to expect that the transport process can almost be ignored. Therefore, the solutions decay to the constant equilibrium states more easily and more quickly.

Similarly, we can observe differences between Theorems 3.4 and 3.6. The former describes the local asymptotic stability of $E_{4}$ and $E_{5}$ in $L^{2}$ norm, while the latter states the global asymptotic stability of $E_{4}$ and $E_{5}$ in the maximum norm, of course, where the additional condition $\sigma>0$ is needed as well.

Third, for the Dirichlet problem, the results are distinct from those of either the O.D.E. problem or the Neumann problem.

We know that the trivial equilibrium solution $(0,0,0)$ must be unstable for the O.D.F. problem and the homogeneous Neumann problem. However, since the homogeneous Dirichlet boundary condition means that none of the three species can exist on the boundary of their habitat, the diffusion mechanism is harmful to the existence of the populations. Obviously, the predator can not be alive alone. So Theorem 4.3 says that all populations go to extinction whenever both $-d_{1} \lambda_{0}+\gamma$ and $-d_{2} \lambda_{0}+\alpha$ are negative, e.g., both diffusions of $u_{1}$ and $u_{2}$ are too strong, or their habitat is too small.

In order to avoid the extinction of all species, the habitat $\Omega$ must be large enough (hence $\mu$ be small enough) or the diffusion of $u_{1}$ or $u_{2}$ not be too strong. Theorems 4.4 and 4.5 give two conditions under which $u_{1}$ or $u_{2}$ can 
be alive alone forever, respectively. It is easy to understand that any equilibrium solutions for the Dirichlet problem cannot be nonzero constant states which do not satisfy the homogeneous Dirichlet boundary condition. The two equilibrium solutions described in Theorems 4.4 and 4.5 are positive functions of spatial variables in $\Omega$. But, due to the homogeneous Dirichlet boundary condition, the closer to the boundary, the smaller is the population density of the stable states.

We note, as well, that the condition of Theorem 4.4 (i.e., $u_{1}$ goes to a stable positive state while $u_{2}$ and $u_{3}$ go to extinction as time tends to the infinity) is weaker than that of Theorem 4.5 (i.e., $u_{2}$ is alive alone forever). This is because the prey $u_{2}$ is fed on by the predator $u_{3}$, while the existence of the mutualist $u_{1}$ is not influenced directly by $u_{3}$.

The study of general equilibrium solutions and their stability (i.e. the cases of coexistence of two or all three species) under the Dirichlet boundary condition is interesting. But, due to the difficulties of the corresponding analysis, we do not deal with it here.

\section{APPENDIX}

The main lemmas cited in this paper are proved in this Appendix. The reader is referred to [1] and [11].

Proof of Lemma 3.1. Suppose $q \geqslant 2$. Then

$$
\begin{aligned}
\frac{d}{d t} \int_{\Omega} u_{3}^{q} d x & =\int_{\Omega} q u_{3}^{q-1} \frac{\partial u_{3}}{\partial t} d x \\
& =\int_{\Omega} q d_{3} u_{3}^{q-1} \Delta u_{3} d x+\int_{\Omega} q u_{3}^{q}\left(-s+\frac{c \beta u_{2}}{1+m u_{1}}\right) d x \\
& \leqslant-\frac{4(q-1)}{q} d_{3} \int_{\Omega}\left(\nabla u_{3}^{q / 2}\right)^{2} d x+q(-s+c \beta M) \int_{\Omega} u_{3}^{q} d x
\end{aligned}
$$

where the constant $M<\infty$. By the Nirenberg-Gagliardo inequalities [16], we have

$$
\int_{\Omega} u^{q} d x \leqslant \varepsilon \int_{\Omega}\left(\nabla u_{3}^{q / 2}\right)^{2} d x+C \varepsilon^{-m}\left(\int_{\Omega} u_{3}^{q / 2} d x\right)^{2}
$$

for $0<\varepsilon<1$, where $m>n / 2, C=C(\Omega, m)$. Hence

$$
\begin{aligned}
-\frac{4(q-1)}{q} d_{3} \int_{\Omega}\left(\nabla u_{3}^{q / 2}\right)^{2} d x \leqslant & -\frac{4(q-1)}{q} d_{3} \varepsilon^{-1} \int_{\Omega} u_{3}^{q} d x \\
& +\frac{4(q-1) C}{q} d_{3} \varepsilon^{(m+1)}\left(\int_{\Omega} u_{3}^{q / 2} d x\right)^{2} .
\end{aligned}
$$


Take $\varepsilon>0$ sufficiently small, such that

$$
-\frac{4(q-1)}{q \varepsilon} d_{3}+q(-s+c \beta M)=-\delta<0 .
$$

Clearly, $\varepsilon \leqslant O\left(q^{-1}\right)$. Thus

$$
\frac{d}{d t} \int_{\Omega} u_{3}^{q} d x \leqslant-\delta \int_{\Omega} u_{3}^{q} d x+\frac{4(q-1) d_{3} C}{q \varepsilon^{m+1}}\left(\int_{\Omega} u_{3}^{q / 2} d x\right)^{2},
$$

and hence

$$
\int_{\Omega} u_{3}^{q} d x \leqslant C_{1} q^{m \cdot 1}\left\|u_{3}\right\|_{H^{4 / 2}(\Omega)}^{4}+C_{2}<\infty
$$

where $C_{1}$ and $C_{2}$ are some positive constants. We need to find a constant $K$ such that

$$
\left\|u_{3}\right\|_{1,4(\Omega)} \leqslant K
$$

for $t \geqslant 0$ and $q=p \cdot 2^{v}, N=1,2, \ldots$.

If. for any $q=p \cdot 2^{N}$,

$$
\sup _{t \geqslant 0} C_{1} q^{m+1}\left\|u_{3}\right\|_{1 \%(\Omega)(\Omega)}^{t} \leqslant C_{2}
$$

then

$$
\int_{\Omega} u_{3}^{p \cdot 2^{v}} d x \leqslant 2 C_{2}
$$

i.e., there exists a constant $\tilde{K}_{1}$ independent of $q$, such that

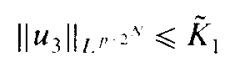

for $t \geqslant 0$ and $N=1,2, \ldots$. Otherwise,

$$
\begin{aligned}
\left\|u_{3}\right\|_{L^{n-2}} x & \leqslant\left(2 C_{1}\right)^{1 / q}\left(q^{m+1}\right)^{1 / 4} \sup _{t \geqslant 0}\left\|u_{3}\right\|_{L^{n} \cdot 2^{*}} 1 \\
& \leqslant \prod_{i=0}^{N-1}\left(2 C_{1}\right)^{2^{2 / q}}\left[\left(\frac{q}{2^{i}}\right)^{m+1}\right]^{2^{i} / q} \sup _{t \geqslant 0}\left\|u_{3}\right\|_{L^{n}} .
\end{aligned}
$$

Since sup so $_{t}\left\|u_{3}\right\|_{t^{\prime}}$ is bounded due to the assumption of the lemma and the 
fact that

$$
\begin{aligned}
\prod_{i=0}^{N-1} & \left(2 C_{1}\right)^{2^{\prime} / q}\left[\left(\frac{q}{2^{i}}\right)^{m+1}\right]^{2^{\prime} / q} \\
& =\prod_{i=0}^{N-1}\left[\left(2 C_{1} p^{m+1}\right)^{1 / p}\right]^{1 / 2^{N}}\left[\prod_{i=0}^{N-1}\left(2^{N-i}\right)^{1 / 2^{N-1}}\right]^{(m+1) / p} \\
& <\left\{\prod_{k=0}^{\infty}\left[\left(2 C_{1} p^{m+1}\right)^{1 / p}\right]^{1 / 2^{k}}\right\}\left\{\prod_{k=0}^{\infty}\left(2^{k}\right)^{1 / 2^{k}}\right\}^{(m+1) / p} \leqslant \text { const }<\infty,
\end{aligned}
$$

the lemma is proved.

Proof of Lemma 3.2. From the system (1.2), we know that

$$
\begin{aligned}
c \frac{\partial u_{2}}{\partial t}+\frac{\partial u_{3}}{\partial t} & =c d_{2} \Delta u_{2}+d_{3} \Delta u_{3}+c \alpha u_{2}\left(1-\frac{u_{2}}{K}\right)-s u_{3} \\
& \leqslant c d_{2} \Delta u_{2}+d_{3} \Delta u_{3}-s\left(c u_{2}+u_{3}\right)+P,
\end{aligned}
$$

where $P$ depends on $\sup _{t \geqslant 0}\left\|u_{2}\right\|_{I^{\infty}(\Omega)}$. Integrating the inequality on $\Omega$ and using boundary value condition (1.4), we have

$$
c \int_{\Omega} \frac{\partial u_{2}}{\partial t} d x+\int_{\Omega} \frac{\partial u_{3}}{\partial t} d x \leqslant-s\left(c \int_{\Omega} u_{2} d x+\int_{\Omega} u_{3} d x\right)+P \operatorname{mes}(\Omega) .
$$

Therefore

$$
\sup _{t \geqslant 0} \int_{\Omega} u_{3} d x \leqslant \sup _{t \geqslant 0}\left(c \int_{\Omega} u_{2} d x+\int_{\Omega} u_{3} d x\right) \leqslant \text { const. }
$$

This proves the lemma.

Proof of Lemma 4.3. We know that

$$
K^{*}=\max \left\{\operatorname{Re} \lambda: \lambda \in \sigma\left(A_{2}\right) \cup \sigma\left(A_{3}\right)\right\}<0 .
$$

By Lemma 4.2, we need only show that there exist $\theta^{*} \in(0, \pi / 2)$ and $\gamma^{*}>0$ such that $\overline{A_{1}}(\mu)$ does not have zero as an eigenvalue whenever $\mu \in S^{*}$.

Let $\eta(\mu)$ be an arbitrary eigenvalue of $\bar{A}_{1}(\mu), \varphi(\mu)$ be the corresponding eigenfunction, $\varphi(\mu) \geqslant 0$, and $\|\varphi\|_{L^{2}}=1$. Put

$$
\begin{aligned}
\mu_{1} & =\operatorname{Re} \mu, & \mu_{2} & =\operatorname{Im} \mu, \\
\eta_{1}(\mu) & =\operatorname{Re} \eta(\mu), & \eta_{2}(\mu) & =\operatorname{Im} \eta(\mu) .
\end{aligned}
$$


Then

$$
\begin{aligned}
& \overline{A_{1}}(\mu) \varphi(\mu)=\eta(\mu) \varphi(\mu), \\
& \eta(\mu)=\left\langle\overline{A_{1}}(\mu) \varphi(\mu), \varphi(\mu)\right\rangle \\
& \quad=\left\langle\left(A_{1}-\frac{\gamma u_{1}^{*}}{L_{0}}\right) \varphi(\mu), \varphi(\mu)\right\rangle+\left\langle-\frac{\gamma u_{1}^{*}}{L_{0}} \varphi(\mu), \varphi(\mu)\right\rangle-\mu,
\end{aligned}
$$

and hence

$$
\begin{aligned}
& \eta_{1}(\mu)=\left\langle\left(A_{1}-\frac{\gamma u_{1}^{*}}{L_{0}}\right) \varphi(\mu), \varphi(\mu)\right\rangle+\left\langle-\frac{\gamma u_{1}^{*}}{L_{0}} \varphi(\mu), \varphi(\mu)\right\rangle-\mu_{1}, \\
& \eta_{2}(\mu)=-\mu_{2} .
\end{aligned}
$$

We know from (4.11) that $u_{1}^{*}$ satisfies

$$
\begin{gathered}
\left(A_{1}-\frac{\gamma u_{1}^{*}}{L_{0}}\right) u_{1}^{*}=0, \quad x \in \Omega, \\
\left.u_{1}^{*}\right|_{\partial \Omega}=0 .
\end{gathered}
$$

(A.2) means that the second order elliptic operator $A_{1}-\gamma u_{1}^{*} / L_{0}$ has the positive eigenfunction $u_{1}^{*}(x) \in H_{0}^{1}(\Omega)$ with zero eigenvalue. It follows that [11]

$$
\left\langle\left(A_{1}-\frac{\gamma u_{1}^{*}}{L_{0}}\right) \psi, \psi\right\rangle \leqslant 0 \quad \text { for any } \quad \psi \in H_{0}^{1}(\Omega)
$$

Thus,

$$
\eta_{1}(\mu) \leqslant\left\langle-\frac{\gamma u_{1}^{*}}{L_{0}} \varphi(\mu), \varphi(\mu)\right\rangle-\mu_{1}<0 \quad \text { for any } \quad \mu_{1} \geqslant 0
$$

In view of $\eta_{1}\left(0, \mu_{2}\right)<0$ and the continuous dependence of $\eta(\mu)$ on $\mu$, we know that there exist $C^{*}>0$ and $\alpha^{*}>0,-\alpha^{*} \in\left(K^{*}, 0\right)$, such that $\eta_{1}\left(\mu_{1}, \mu_{2}\right)<0$ whenever

$$
\mu \in\left\{\mu \in \mathbb{C}: \mu \in \rho\left(A_{2}\right) \cap \rho\left(A_{3}\right), \operatorname{Re} \mu \in\left(-\alpha^{*}, 0\right),|\operatorname{Im} \mu|<C^{*}\right\} .
$$

We note that $\left|\eta_{2}\left(\mu_{1}, \mu_{2}\right)\right|=\left|\mu_{2}\right| \geqslant C^{*}>0$ when $\left|\mu_{2}\right| \geqslant C^{*}$, so $\overline{A_{1}}(\mu)$ cannot have zero as an eigenvalue if

$$
\mu \not \subset\left\{\mu \subset \mathbb{C}: \mu \subset \rho\left(A_{2}\right) \cap \rho\left(A_{3}\right), \operatorname{Rc} \mu<\alpha^{*},|\operatorname{Im} \mu|<C^{*}\right\} .
$$


Taking

$$
\theta^{*} \in\left(0, \arctan \frac{\alpha^{*}-\gamma^{*}}{C^{*}}\right) \text { and } \gamma^{*} \in\left(0, \alpha^{*}\right)
$$

we complete the proof of Lemma 4.3.

The author wishes to express his thanks to Professor J. Smoller and Professor L. Hsiao for their encouragement and help.

\section{REFERENCES}

1 N. Alikakos, An application of the invariance principle to reaction-diffusion equations. J. Differential Equations 33:201-225 (1979).

2 K. Chueh, C. Conley, and J. Smoller, Positively invariant region for systems of nonlinear diffusion equations, Indiana Unie. Math. J. 26:373-392 (1977).

3 E. Conway, D. Hoff, and J. Smoller, Large time bchavior of solutions of systems of nonlinear reaction-diffusion equations, SIAM J. Appl. Math. 35:1-16 (1978).

4 R. Courant and D. Hilbert, Methods of Mathematical Phisics, Vol. 1. Wiley-Intersicience. New York, 1962.

5 J. P. G. Ewer and L. A. Peletier, On the asymptotic behavior of solutions of semilinear parabolic equations, SIAM J. Appl. Math. 28:43-53 (1975).

6 P. Fife, Mathematical Aspects of Reacting and Diffusion Systems, Springer, New York, 1979.

7 D. Henry, Geometric Theory of Semilinear Parabolic Equations. Springer, New York, 1980.

8 L. Hsiao, Y. Su, and Z. P. Xin. On the asymptotic behavior of solution of a reaction-diffusion system: A two predator-one prey model, to appear.

9 L. Hsiao, Y. Su, and Z. P. Xin, Asymptotic behavior of solution of a reaction-diffusion system: Competing predators in a chemostat, to appear.

10 L. Markus. Asymptotically autonomous differential system, in Contrihutions to the Theory of Nonlinear Oscillations, Vol. 3, Ann. Math. Studies, No. 36, Princeton, U.P.. Princeton, 1956, pp. 165-198.

11 D. de Mottoni and A. Tesei, Asymptotic stability results for a system of quasilinear parabolic equations, Applicable Anal. 9:7-21 (1979).

12 C. V. Pao, On nonlinear reaction-diffusion systems, J. Math. Anal. Appl. 87:165-198 (1982)

13 B. Rai, H. I. Freedman, and J. F. Addicott, Analysis of three species model of mutualism in predator-prey and competitive systems, Math. Biosci. 65:13-50 (1983).

14. J. Serrin, A remark on the preceding paper of Amann, Arch. Rational Mech. Anal. 44:182-186 (1972).

15. J. Smoller, Shock Waves and Reaction-Diffusion Systems, Springer, New York, 1983.

16. E. M. Stein, Singular Integrals and Differentiability Properties of Functions, Princeton U.P.. Princeton, 1970. 